\title{
XVI.
}

Aus der med. Klinik des Herrn Professor Ribbing zu Lund (Schweden).

\section{Ein Fall von Paralysis agitans, mit verschiedenen Myxödemsymptomen combinirt.}

Studien nnd Gedanken über die Pathogenese der Paralysis agitans. ${ }^{1}$ )

Von

\section{Herman Lundborg.}

(Mit Tafel III, IV und einer Abbildung im Text.)

\section{Einleitung.}

Seit anderthalb Jahren gebe ich mich damit ab, eine grosse Bauernfamilie aus der Provinz Blekinge zu studiren, eine Familie, in welcher mehrere Nervenfälle sehr sonderbarer Art vorgekommen sind. Obgleich meine Untersuchungen bei weitem nicht beendet sind, glaube ich doch schon Verschiedenes gewonnen za haben.

Durch Studien am Orte und in der Literatur ist es mir klar geworden, dass eine verhältnissmässig nur geringe Zahl jener Krankheitsfälle, die ich dortselbst näher beobachtet habe, in der Literatur beschrieben worden ist, und zwar unter dem Namen "familiäre Myoklonie".

Durch die Liebenswürdigkeit, die mir von Seiten des Karolinischen Instituts in Stockholm zu Theil geworden ist, wurde ich in die glückliche Lage versetzt, behufs näherer klinischer Untersuchung zwei Fälle nach Stockholm herauf zu bekommen. In Folge der Erfahrungen betreffs der Symptomatologie, welche ich damals gemacht habe, stieg in mir der Verdacht auf, die Myoklonie sei eine Krankheitserscheinung, welche durch gewisse Veränderungen der Schilddrüse bedingt werde. Auf die Gründe einer derartigen Auffassung kann ich hier jetzt nicht näher eingehen, da es zu viel Zeit in Anspruch nehmen würde; ich verspreche aber bei einer anderen Gelegenheit auf diese Sache zurückzukommen. Kurz, ich bin dahin gekommen, die Myoklonie als die Folge einer Erkrankung der-Thyreoidea aufzufassen. Zu bemerken

1) Bei der Sitzung des „Vereins der Aerzte in Lund“ den 31. Oct. 1899 vorgetragen. 
ist jetzt, dass in der nämlichen Familie nicht weniger als fünf Fälle von Paralysis agitans vorgekommen sind, welcher Umstand natürlich gleich meine Aufmerksamkeit auf sich lenkte. Ich fing an, anf die beiden Krankheiten näher einzugehen und deren Symptome mit einander zu vergleichen, und konnte mich der Ansicht nicht erwehren, dass eine gewisse, und zwar recht grosse Uebereinstimmung unter den beiden Krankheiten bestehe. Demgemäss war ich so kühn, die Consequenzen auch noch auf die Pathogenese auszudehnen, indem ich bei mir selbst so dachte: Ist die familiäre Myoklonie, so wie ich sie in Blekinge habe auftreten sehen, eine Schilddrüsenkrankheit, so ist allem Anscheine nach die Paralysis agitans dies auch. Diese meine Auffassung theilte ich dem Herrn Prof. Lennmalm in Stockholm im März v. J. mit und hörte ich dann zu meiner grossen Freude von ihm, dass Möbius Ende 1898 sich in einerlei Richtung ausgesprochen habe. Herr Prof. Lennmalm hat die grosse Liebenswürdigkeit gehabt, mir jene Stelle nachzuweisen, wo Möbius eine derartige Ansicht geäussert hat. Ich kann nicht umhin, meine Herren, Ihnen von seinem diesbezüglichen kurzen Ausspruch Mittheilung zu machen, welcher sich in seinem Werke "Neurologische Beiträge" (Vermischte Aufsätze, V. Heft, S. 19), vom Jahre 1898 wiederfindet. Da schreibt er:

„Nach Analogie möchte man annehmen, dass der Paralysis agitans eine Stoffwechselstörung zu Grunde liege, dass ein abnormes Stoffwechselproduct oder das Fehlen eines nöthigen Stoffes ihre Ursache sei. Reizerscheinungen, die ohne gröbere anatomische Läsionen bestehen, deuten auf chemische Einwirkungen. Ich habe früher einmal (Combination von Morbus Basedowii und Paralysis agitans. Memorabilien 1883, 3. Heft) auf die Züge aufmerksam gemacht, die die Paralysis agitans und die Basedow'sche Krankheit gemein haben: Zittern (wenn auch verschiedener Art), Hitzegefühl, Neigung zum Schwitzen, Gefühl der Unruhe, Pulsbeschleunigung, Einfluss von Gemüthsbewegungen. Haben wir früher von der merkwürdigen Thätigkeit der Schilddrüse, der Thymus, des Hirnanhangs nichts gewusst, so ist es wohl möglich, dass auch noch andere Drüsen eine Rolle im Stoffwechsel spielen, von der wir keine Ahnung haben.

Wenn nun bei manchen Leuten die ,quästionirte' Drüse dem Altersschwunde verfiele, so würde auf ihre Atrophie die Paralysis agitans folgen können, wie das Myxödem auf die der Schilddrüse. Diese Hypothese scheint mir vernünftiger zu sein als das unaufhörliche Herumsuchen im Rückenmark, ob nicht da oder dort ein Stellchen verhärtet sei oder gar ein paar Blutgefässe entartet seien."

Hieraus erhellt, dass Möbius hinsichtlich des Wesens der Paralysis agitans einen weit positiveren Standpunkt einnimmt, als Jemand 
vor inm gethan hat und dass er die Richtung andeutet, in welcher die Untersuchungen fortgesetzt werden missen.

Aus diesem Grunde habe ich sowohl Herrn Prof. Lennmalm als Herrn Prof. Edgren darum ersucht, auf ihren resp. Abtheilungen Versuche mit Thyreoidinbehandlung in Fällen von Paralysis agitans anstellen zu dürfen, welche Bitte mir gewährt wurde. Während des Frühlings wurde also in jeder Klinik je ein Fall eine Zeit lang auf diese Weise behandelt, ohne dass man doch etwas Bestimmtes hieraus folgern konnte, auf Grund der verhältnissmässig kurzen Zeit, in der wir Gelegenheit hatten die Fälle zu beobachten.

Einen ähnlicben, ziemlich weit vorgeschrittenen Krankheitsfall behandelte ich in Blekinge während des Frühlings und Sommers mit Tabletten, ohne dass aber irgend welche günstige Einwirkung wahrgenommen werden konnte; eher möchte ich das Gegentheil behaupten. Sollten auch die Ergebnisse nicht so glänzend werden, halte ich es doch für sehr nöthig, dass noch mehr Versuche dieser Art gemacht werden, um grösseres Licht über die Frage zu werfen. Vielleicht, m. H., giebt es einige unter Ibnen, die jetzt diese Einwendung machen möchten, dass die nur sehr mässige, manchmal sogar ungünstige Einwirkung der Thyreoidinbehandlung auf die Kranken mit meiner Auffassung betreffs der Pathogenese der Paralysis agitans im Widerspruch stehe, aber ich bitte solchenfalls diese Herren, zu beachten, dass betreffs der Basedow'schen Krankheit massenhafte Versuche dieser Art mit sehr schlechtem Erfolg gemacht worden sind und immer noch gemacht werden; trotzdem schreibt man sehr allgemein den Veränderungen der Glandula thyreoidea eine sehr grosse Rolle in Bezug auf jene Krankheit zu.

Ohnedies steht es fest, dass es verschiedene sehr gut entwickelte Basedow-Fälle giebt, die ohne Struma verlaufen.

Sie können sich leicht vorstellen, meine Herren, wie gross meine Ueberraschung sein musste, so bald eine so zu sagen lebende Illustration meiner Hypothese anzutreffen. Gleich am ersten Tag, als ich die Runde hier in der Klinik ${ }^{1}$ ) mache, werde ich eine Frau gewahr, die einige Tage vorher unter der Diagnose „Paralysis agitans nebst trophoneurotischen Störungen" aufgenommen worden ist. Ihr Aeusseres befremdete mich gleich, indem sie in ziemlich hohem Grade myxödematös erschien. Ich erlaube mir, sie den geehrten Herren vorzuführen, damit Sie selbst urtheilen können. Nachher will ich den Krankenbericht und die Epikrise durchgehen.

1) In der Abtheilung des Herrn Prof. Ribbing. 
Krankenbericht. Caroline D., Wittwe, 54 Jahre alt.

Anamnese. Der Vater der Patientin hat das Alter von 83 Jahren erreicht und soll stets gesund gewesen sein. Eine seiner Schwestern, welch $\theta$ mit 69 Jahren starb, litt am Ende ihres Lebens mehrere Jahre an Tremor im Körper, besonders in den Armen. Man hält es für wahrscheinlich, dass sie an derselben Krankheit wie die Patientin oder wenigstens an einer jener sehr ähnlichen gelitten hat. Die Mutter der Pat. soll von Kindheit an in der rechten Gesichtshälfte und im Halse derselben Seite Spasmus gehabt haben. Im späteren Theile ihres Lebens litt sie nach Mittheilungen der Pat. an „Tic douloureux“. Einen ebensolchen Tic soll auch ihre Mutter (die Grossmutter der Pat.) gehabt haben. Sonst weiss die Pat. nichts von etwaigen schwereren Nerven- oder Geisteskrankheiten ihrer Vorfahren.

Die Pat. ist die zweitälteste von 10 Geschwistern. (Ausser diesen hat die Mutter noch ein todtgeborenes Kind zur Welt gebracht, das letzte in der Reihe.) Fünf Geschwister sind gestorben. Ein Bruder starb im Alter von 2 Jahren an einer unbekannten Krankheit, ein zweiter Bruder starb als geisteskrank in der Irrenanstalt zu Lund, 45 Jahre alt. (Er wurde dortselbst unter der Diagnose Dementia paralytica gepflegt.) Eine Schwester, welche das Alter von 42 Jahren erreichte, soll nach ärztlichem Bericht Rückenmarksleiden gehabt haben, eine andere Schwester starb an Brustkrebs. Die eine der noch lebenden Schwestern der Patientin ist ,in der Brust krank", eine zweite hat Tic douloureux, wie die Mutter. Die zwei übrigen Geschwister (Brüder) sind am Leben und beide „dick und stark".

Die Pat. hat den Partus dreimal durchgemacht. Die Kinder leben und sind gesund.

Die Pat. soll in jüngeren Jahren ziemlich schwächlich gewesen sein; nach Angaben des Arztes, welcher die Pat. vor der Aufnahme gepflegt hat, ist sie immer „nervös" gewesen. Die Menstruation in der Jugend mit gewissem Unbehagen, wie Schmerz u. dgl. vereinigt, später nicht. Dieselbe soll stets regelmässig gewesen sein und hat sich bis vor drei Monaten eingefunden, wo sie zum ersten Male ausblieb.

Pat. wurde mit der Zeit sehr beleibt. Vor 13 Jahren litt sie erheblich an ..Gallensteinbildung". Die Haut wurde hochgradig gelb gefärbt, und schmerzhafte Kolikanfälle stellten sich wiederholt ein. Der gerufene Arzt verordnete eine Abmagerungscur, welcher sie sich auch 3 Wochen lang unterwarf. Dann musste sie aufhören, weil sie sich zu schwach fiihlte. Mehrere Jahre hindurch wiederholten sich später die Kolikanfälle. Während der letzten 8 Jahre hat die Pat. von diesem Uebel nichts gemerkt.

Thre jetzige Krankheit hat sich vou jenem Zeitpunkte an allmählich entwickelt. Im Jahre 1891 empfand die Pat. nämlich zuerst ein leichtes Zittergefühl im linken Ringfinger und in den Zehen des linken Fusses. Hieran reihten sich bald Schmerzen in der linken Hand, im Unterarme und Ellenbogen und in der Wade derselben Seite. Um die Schmerzen zu lindern, pflegte die Pat. so lange auf dieser Hand zu sitzen, bis sie gefühllos wurde. Binnen Kurzem verbreitete sich der Tremor von den peripher gelegenen Theilen der linken Extremitäten allmählich aufwärts. Folgendes Jahr, 1892, zitterte schon der ganze linke Arm, bezw. Bein. Darauf wurde die linke Schulter sowie benachbarte Theile der Brust befallen. Nach 
einigen Jahren stellte sich der Tremor auch noch in der rechten Seite ein, wo er sich allmählich, genau wie vorher auf der linken Seite, längs der Extremitäten nach oben fortpflanzte.

Auch hier fühlte die Pat. bisweilen Schmerzen. Der Tremor dehnte sich fortwährend aus und ging auf die Brust- und Bauchmuskeln über, dann auf Hals, Zunge, Unterkiefer und Lippen. Das Gesicht im Uebrigen blieb verschont.

Gleichzeitig mit Auftreten dieser Muskelunruhe fing Pat. an sich körperlich steif und matt zu fühlen. Die Bewegungen wurden langsamer, und die Pat. immer regungsloser. Seit mehreren Jahren hat sich Pat. im Bette von selbst nicht umdrehen können. Die meiste Zeit hat sie während der letzten Jahre in einem Sessel, in vornüber gebückter und hockender Stellung zugebracht. Das letzte halbe Jahr hat sie sogar im Sessel geschlafen. Der Kopf ist während der letzten Monate so tief herabgesunken, dass das Kinn an die Brust gestossen hat, was auf der unteren Fläche des Kinnes Decubitus zur Folge gellabt hat.

Bis vor einem Jahre hat die Pat. ohne Hülfe, wenn auch langsam, gehen können. Seit jener Zeit hat man sie beim Gehen unterstützen miissen. Die Sprache hat sich geändert. Die Pat. hat angefangen, langsamer und mit tieferer Stimme zu sprechen und ermüdet schnell. Die Intelligenz, namentlich das Gedächtniss, scheint ihr nicht in etwaigem höheren Grade herabgesetzt zu sein.

Dass ihr Gemüth sich aber geändert hat, giebt sie zu. Sie ist so traurig und weinerlich geworden. „Früher konnte ich reden, lachen und bei guter Laune sein", sagt sie, „das kann ich jetzt nicht mehr." Ausserdem wird sie öfters von einer inneren Unruhe gequält.

Das Haar, das früher so dick war, ist sehr dünn geworden. Auf die Frage, ob sie etwa eine grössere Veränderung seit Anfang der Krankheit an ihrem Aussehen wahrgenommen habe, antwortet sie: „Ja, die Augen sind kleiner geworden, die Gesichtszüge rauher, Nase und Lippen grösser, so dass ich mich gegen früher nicht mehr erkenne."

Schon vor einigen Jahren bemerkte die Pat. an verschiedenen Stellen des Körpers (Füssen, linker Hand, Gegend des linken Auges) ein plötzlich auftretendes und dann wieder verschwindendes Oedem. Das Oedem in der linken Augengegend zeigte sich oft des Morgens am deutlichsten. Vor etwa 3 Monaten fingen die Unterschenkel und Füsse an, beträchtlich zu schwellen.

Kurz vor der Aufnahme brach die Haut am linken Unterschenkel an mehreren Stellen anf, und eine klare Flüssigkeit begann hervorzusickern. Dieser Zustand dauerte ein paar Tage an. Dessen ungeachtet nahm das Bein an Dicke nicht ab. Bald heilten die Hautwunden.

Der Appetit ist stets sehr gut gewesen, vor einigen Jahren sogar hatte sie mitunter Heisshunger. Ueber schwereren Durst hat die Pat. nie klagen können. Sie war, wie oben erwähnt worden ist, bis vor einigen Jahren sehr wohlgenährt, wo sie allmählich zu magern anfing. Stuhlgang stets regelmässig. In der letzten Zeit hat die Pat. gemerkt, dass es ihr mitunter schwer fällt, den Urin zu halten. Derselbe ist aber bis jetzt noch nie wider Willen gelassen worden. Der Unterkiefer hat angefangen. eine andere Lage gegen früher einzunehmen. Er ist nach hinten und ein wenig nach rechts hinüber gerïckt. Ausserdem ist es der Pat. so vorgekommen. als 
ob die Zähne sich von einander getrennt hätten, indem deutliche Lücken, besonders zwischen den Schneidezähnen, entstanden sind. Voriges Jahr litt Pat. eine Zeit lang an Parästhesie auf der rechten Seite des Halses und im Nacken. Es dünkte ihr oft, als krieche ein Wurm ihr auf der Haut. Seit mehreren Jahren hat die Pat. in der linken Gesichtshälfte eine Empfindung; als sei sie abgestorben. Seit einigen Jahren treten auch noch von Zeit zu Zeit ,förmliche Krampfanfälle auf, so dass man die Pat. halten muss", lant Bericht des früheren Arztes. Dieselben stellten sich oft früh Morgens gleich nach dem Erwachen ein, bisweilen auch am Tage, wenn Pat, sehr traurig geworden war oder einen Jchreck bekommen hatte $u$. s. w. Was die Anfälle betrifft, liefert die Pat selost folgende Mittheilungen:

Der ganze Körper wurde während einiger Minuten von einem starken Kältegefühl durchdrungen, einem wahrem, ,Nervenfrost", wie es einer ihrer früheren Aerzte genannt hat. Zu gleicher Zeit hatte die Pat. ofters ganz unbehagliche, nicht zu beschreibende Empfindungen. „Es kam mir ungefähr so vor", erzählt sie, ,als wenn man mittelst eines Griffels einen schrillenden scharfen Ton an einem Fenster oder einer Steintafel hervorbringt". Nachdem der Frost anfgehört hatte, gerieth der Körper in Schweiss, wobei diejenigen Muskeln, welche zittern, in heftigere Bewegungen versetzt wurden, grössere, gleichzeitige rhythmische Excursionen machend. Ausserdem stellten sich Angst und Athemnoth ein nebst einem starken Schmerzgefühl im Körper, besonders in den Armen.

In der letzten Zeit haben sich die Anfälle sehr gemässigt, so dass nunmehr nur ausgesprochene Zittererscheinungen nebst Schwitzen, Angst und Schmerz sich zu gewissen Zeiten einstellen. Das Gefühl der Kälte und des Schauders hat immer mehr nachgelassen und hat einem fast steten Hitzegefühl den Platz geräumt, Einige Wochen vor der Aufnahme nahm die Pat. eines Morgens beim Erwachen wahr, dass sie die linke Hand nicht mehr bewegen konnte. Pat. hat die Gewohnheit, im Schlafe den Kopf an diesen Arm zu lehnen, welcher von der Seitenlehne des Stuhles gestiitzt wird. Die Hand hing schlaff herunter, in hochgradiger Flexionsstellung; sie war kalt und gefühllos. Es dauerte aber nicht lange, bis diese Erscheinungen wieder rückgängig wurden.

Die Pat. wurde am 23. Aug. 1899 in die med. Klinik aufgenommen. Es wurden Jodkalium ( $1 \mathrm{~g}$ dreimal täglich) und Faradisation sowie Chloral, zum nächtlichen Gebrauch, verordnet.

Tagesbemerkungen. 8. Sept. 1899. Das Jodkalium wird ansgesetzt, weil Pat. einen andauernden Schnupfen bekommen hat.

Heute Nacht überkam die Pat. ein recht schwerer Anfall, nachdem sie eine Weile geschlafen hatte. Sie wurde von einer schweren inneren Unruhe gepackt. Der gewöhnliche Tremor ging in ein wahres Schütteln über (klonische, gleichzeitige Zuckungen in ziemlich schnellem Rhythmus, welche besonders in den Armen auftraten). Körper zusammengekanert, wie gewöhnlich. Die Pat. war sehr erröthet und schwitzte stark. Die Thränen liefen ihr herunter, und von der Nase fielen grosse l'ropten einer klaren Flüssigkeit. Pat. ächzte und stöhnte und litt allem. Anscheine nach sehr. Das Sensorium ein wenig benommen; Pat. sprach mitunter unklar. Sie erkannte mich doch, als ich zur Stelle kam, phantasirte und meinte, ihre letzte Stunde sei gekommen. Sie redete wie mit ihrem verstorbenen Mann, indem sie äusserte: „Ich komme bald, Väterchen". Dann schwatzte sie von 
einem Zuge, der ihre Tochter mitbringen werde u. s. w. Die Pat. wurde aus dem Bett genommen und auf ihren gewöhnlichen Platz im Stuhle gebracht, wonach sie sich einigermassen beruhigte. Sie bat mehrmals, wir möchten sie doch aus dem Stuhle heben und sie einige Schritte gehen lassen, was geschah. Hierdurch empfand sie Linderung. Sie bekam $1 \mathrm{cg}$ Morphin und erhielt die Erlaubniss im Stuhle bleiben zll dürfen. Nach einer Weile schlief sie ein.

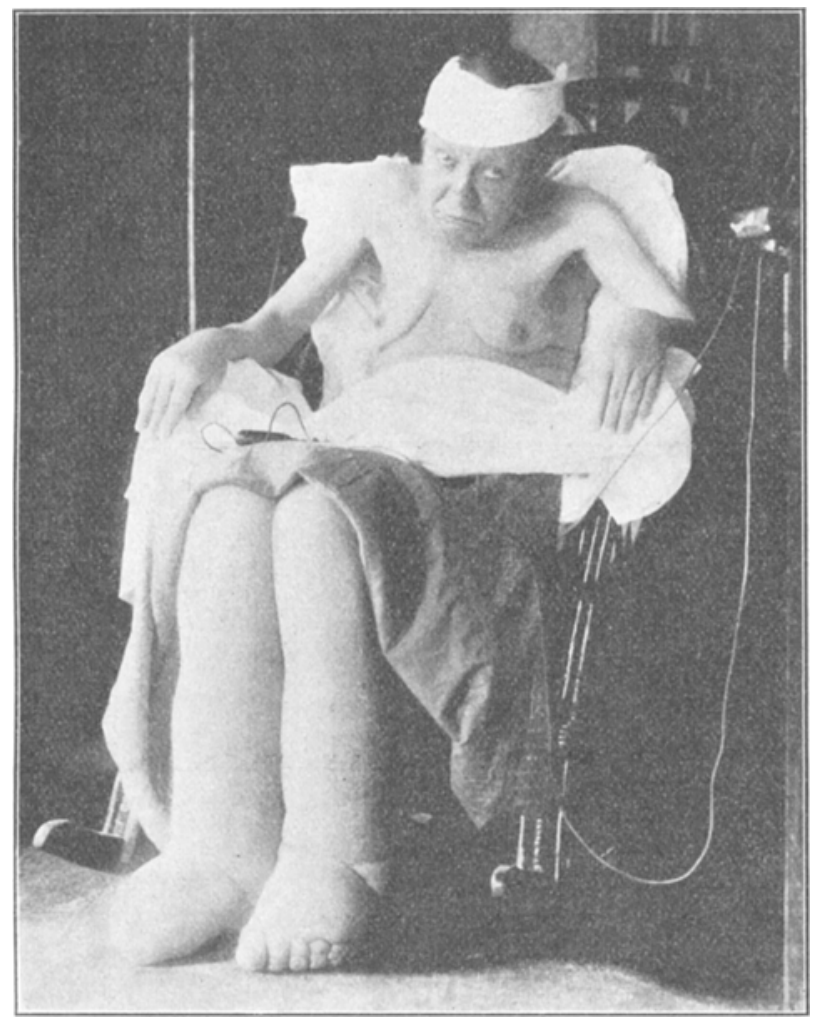

Fig. 1.

Status praesens am 12. Sept. Ihre meiste Zeit bringt die Pat, in einem bequemen Lehnsessel zu, wo sie in Kissen wohl eingebetcet sitzt, welche hinter den Rücken und nnter die Arme placirt worden sind. Die Arme ruhen meistentheils auf den beiden Seitenlehnen.

Die Pat. klagt über grosse Mattigkeit und Steife im Körper, was zur Folge hat, dass sie von selbst höchstens ein paar Bewegungen machen kann.

Ausserdem wird sie von Tremor und einem starken Hitzegefühl nebst Schwitzen heimgesucht. Oft hat die Pat. Schmerzen in verschiedenen Theilen des Körpers. 
Der. Schlaf ziemlich schlecht, weshalb die Pat. für die Nacht öfters beruhigende Mittel erhält ( 1 bis $2 \mathrm{~g}$ Chloral). Während des Schlafes ist keine Muskelunruhe beobachtet worden. afebril.

Der Appetit ist ganz gut. Stuhlgang regelmässig. Die Temperatur

Die Pat. ist blond. Haarwuchs ziemlich schlecht. Die Haare auf dem Kopfe doch gleichmässig vertheilt. Haare an den Angenbrauen, in den Achselhöhlen und an der Scham von hellbrauner Farbe. Die Augen sind matt und blau. Fettpolster ziemlich gering. Die Musculatur nicht stark entwickelt, scheint aber nirgends gerade atrophisch $\mathrm{zu}$ sein.

Hant meist dünn, blass und von normaler Elasticität, mit Ausnahme einiger bestimmter Stellen des Körpers, von denen später die Rede sein wird. Pat. schwitzt mitunter recht erheblich. Dermatographie tritt allenthalben sehr deutlich hervor.

Der Gesichtsausdruck ist stereotyp. Man kann die Pat. doch lächeln machen, wenn man sie dazu auffordert, spontan thut sie es äusserst selten. Im Gesicht spiegeln sich Trübsinn, Ernst und Stumpfsinn ab. Pat. ist sehr weinerlich. Spricht langsam und mit ziemlich tiefer Stimme; hört sich oft furchtbar traurig an.

Intelligenz ziemlich träge. Gedächtniss schwach. Ihr Gemüth ist sehr unruhig und fordert sie nicht so wenig von ihrer Umgebung. Dies thut ihr leid und sie klagt, dass sie so viel Beschwerden verursacht! Sie bittet deswegen oft um Verzeihung und erklärt, dass sie nicht dafür könne, weil die Krankheit sie derart verändert habe.

Die Gesichtshaut weist einen eigenthümlichen Farbenton auf, in dem vorwiegend ein nicht gar zu starkes Roth znm Vorschein kommt, mit einem Stich ins Blaue und auch noch ein klein wenig ins Braune.

An vielen Stellen und zwar an Backen, Nase und Kinn fühlt sich die Haut verdickt und wie infiltrirt an. Die Gesichtszüge sind rauh, die Nase ist dick und stumpf. Die Nasolabialfurchen treten deutlich hervor. Die Lider erscheinen öfters ein wenig angeschwollen. Unterhalb der Augen, insbesondere des linken, hängt die Haut herunter und bildet gewissermassen kleine Säckchen. Lippen gross, ein wenig cyanotisch. Sämmtliche Schneidezähne im Oberkiefer sind noch vorhanden und stehen ziemlich weit von einander getrennt. Unterkiefer etwas nach hinten gezogen; die Pat. ist trotz der grössten Anstrengung nicht im Stande, die Vorderzähne gegen einander zu drücken; die unteren stehen immer dahinter. (Pat. kann den Mund ordentlich aufmachen.) Zunge von normalem Aussehen, nicht vergrössert; zeigt Tremor.

Das Schlucken in keiner Weise beeinflusst. Die Bewegungen der Augen ganz normal.

Was die höheren Sinne betrifft, scheint nichts Besonderes der Erwähnung werth. Die Sensibilität überall normal.

Körperstellung hockend. Der Kopf ist so tief herabgesunken, dass das Kinn zumeist an die Brust stösst. Kopf oft etwas nach rechts rotirt, nach links flectirt. Um dem Decubitus vorzubeugen, ist der Pat. eine breite Binde um die Stirn gelegt, nachher gespannt und am Rücken des Sessels festgemacht worden. Demgemäss wird die Pat. gezwungen, den Kopf bedeutend höher zu halten, als sie es sonst hätte thun können. Sie kann die Binde doch nur von Zeit zu Zeit anhaben, weil dieselbe ihr auf 
die Dauer zu lästig wird. Die Beine lässt sie herabhängen, indem sie die Fusssohlen auf den Boden stïtzt. Pat. ist nicht im Stande, sich von selbst zu erheben, noch kann sie gehen. Stehen kann sie aber, wobei sie die Beine sowohl in Hüft- wie Kniegelenken flectirt hält. Der Rücken bildet eine stark ansgesprochene Rundung, die sich vorwärts über Hals und Nacken fortsetzt. Das Gesicht sieht nach dem Boden.

Die rechte Schulter ragt deutlich über die linke empor. Die Arme sind ungefähr bis zu einem rechten Winkel flectirt.

Stützt man die Pat. an der einen Hand, so vermag sie, wenn zwar langsam und mit kleinen Schritten, zu gehen. Sie steht gern auf, um ab und zu ein paar Schritte zu nehmen; das schwächt, meint sie, verschiedene subjective Symptome.

Lässt man die Pat. in Ruhe, verhält sie sich ziemlich still; man merkt nur einen gleichförmigen und regelmässigen Tremor in den Händen und Unterarmen. Wird sie dagegen in irgend einer Weise beunruhigt, so nimmt der Tremor an Stärke beträchtlich zu, die Bewegungsexcursionen werden grösser, und der Tremor greift auf andere Gebiete des Körpers ïber und zwar anf Beine, Schultern, Brust, Hals, Kopf, Unterkiefer, sowie Kinn und Lippen. Man hört dann die Zähne im Takte des Tremors klappern. Der Schweiss nimmt zu, die Augen werden von Thränen gefüllt, und die Nase tröpfelt. Lässt man die Pat. in Ruhe, so hören diese Erscheinungen sehr bald wieder auf. Der Körper ist sehr rigid, wechselnd je nach dem Orte. Pat. kann von selbst den Kopf nur sehr unbedentend heben; hierbei spannen sich sichtbar mehrere von den Halsmuskeln, vor Allem die beiden Sternocleidomastoidei. Beim Versuch, der Pat. bei dem Heben des Kopfes behülflich zu sein, gelingt dies zum Theil auch, der Kopf leistet aber einen erheblichen Widerstand. Pat. kann den Kopf sehr langsam nach beiden Seiten drehen. Der Hals ist kurz und stark vornüber gebeugt. Die Schultern stehen ungefähr in gleicher Höhe mit den untersten Theilen der Ohren. Der Brustkasten ist in nicht geringem Grade deformirt, zu Folge des steten Druckes des Kopfes gegen den oberen Theil des Sternum.

Das Sternum als Ganzes bildet ungefähr auf der Mitte einen hinten offenen stumpfen Winkel; zu beiden Seiten dieses Winkels ragen zwei starke gleichförmige Ausbuchtungen der Brustwand hervor, an deren Bildung mehrere Rippenknorpel theilnehmen. Gleich unter- und ausserhalb derselben gewahrt man die beiden ziemlich schlaffen Mammae.

Bei der physikalischen Untersuchung der Brustorgane findet man die Lungen emphysematös; im Uebrigen nichts Abnormes. Der Radialpuls recht gut und regelmässig; die Frequenz wechselt zwischen 80-100 pro Minute, beträgt gewöhnlich $84-86$.

Der Bauch weich und nachgiebig; seine Organe scheinen nichts $A b$ normes zu bieten. Der Harn klar, sauer, von gesättigt-gelber Farbe; enthält weder Eiweiss noch Zucker. Spec. Gewicht 1,028. Die Harnmenge pro 24 Stunden wechselt zwischen 900 und $1300 \mathrm{ccm}$. Die Arme und Hände können noch recht gut bewegt werden. Sie sind trotzdem ziemlich rigid, ebenso wie die Brust sehr mager, ein Umstand, der um so mehr in die Augen tritt, als Unterschenkel, Füsse und Kopf so plump sind. Am rechten Arm tritt häufig der Supin. long. mit seinem gespannten Muskelbattche besonders deutlich hervor; ein ähnliches Verhalten ist zuweilen auch am 
linken Arm wahrgenommen worden. In letzterem zeigt sich nunmehr keine Spur der in der Anamnese besprochenen Lähmung. Die rechte Hand vermag Pat. bis zum Nacken zu heben, die linke dagegen nur bis an den Mund. In den Händen und Unterarmen tritt der Tremor öfters mit grosser Deutlichkeit hervor. Die vier ulnaren Finger der linken Hand sind in den Metacarpophalangealgelenken flectirt, in den beiden anderen Gelenken hingegen ein wenig hyperextendirt und zwar stärker im ersten Phalangealgelenk als im zweiten. Die letzte Phalanx des Zeigefingers ist mässig flectirt. Der Daumen ist adducirt, die erste Phalanx befindet sich in Extensionsstellung, die zweite ist in ungefähr rechtem Winkel flectirt. Es ist der Pat. so vorgekommen, als ziehe sich der linke Daumen immer mehr in die Hand hinein. An der rechten Hand wird keine Hyperextension irgend welcher Fingergelenke wahrgenommen. Die Haut an den Armen überall dïnn, kein Oedem. Die Beine sind von den oberen Theilen der Unterschenkel bis zu den Zehen herab gewaltig verdickt, was ans folgenden Maassen erhellt:

\begin{tabular}{|c|c|c|c|c|}
\hline Umkreis & gleich unterhalb der Patella & 34 & $\mathrm{~cm}$ & $34 \mathrm{~cm}$ \\
\hline - & Mitte des Unterschenkels & 42,5 & , & 44 \\
\hline . & Höhe des Malleolus & 38 & " & 39 \\
\hline , & Mitte des Fusses & 34 & 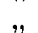 & 35 \\
\hline
\end{tabular}

Zwischen Fuss und Unterschenkel zieht sich beiderseits eine tiefe Hautfurche. Haut an denOberschenkeln dünn und blass, an den Unterschenkeln hingegen dick und fest, von blauroter Farbe; dieselbe schuppt ein wenig and weist hie und da mehrere Risse auf.

Nirgends ist man im Stande, mit dem Finger einen bleibenden Eindruck hervorzubringen. Am linken Unterschenkel zeigen sich mehrere harte Krusten als Merkmale der in der Anamnese erwähnten Hautrisse. An den Fusssoh len keine nennenswerthe Verdickung, um so mehr aber auf den Fussrücken und zwar auf der Mitte derselben. Die Patellarreflexe herabgesetzt. Die Sensibilität in den Beinen ungestört. Die Pat. vermag von selbst die Beine zu strecken, völlig doch nicht. In denselben kommt unter gewöhnlichen Verhältnissen kein Tremor zum Ausdruck, nur während der „Anfälle" stellt er sich ein.

Bei der Blutuntersuchung stellt sich der Hämoglobingehalt (nach Fleischl bestimmt) auf 75 .

19. Sept. Die Pat. fängt heute mit der Tbyreoidinbehandlung an und bekommt täglich eine Tablette (à $33 \mathrm{cg}$ ans Burroughs, Wellcome, London).

10. Oct. Auf der inneren Seite des linken Unterschenkels ist die Hant heute eingerissen, so dass eine kleine blutende Wunde sich gebildet hat. Lapistouchirung. Beide Unterschenkel sowie Füsse haben an Umfang etwas zugenommen. Der Pat. werden feuchtwarme Umschläge, mit verdünntem Liq. acet. alum. durchtränkt, verordnet und bekommen diese ihr wohl.

18. Oct. Die Pat. ist mit ihren Umschlägen sehr zufrieden. Seit einigen Tagen Schnupfen.

19. Oct. Heute Morgen empfand die Pat. während circa einer Stunde ein starkes Kältegefühl im Körper, welche Erscheinung seit Langem nicht 
aufgetreten ist. Während der Morgenrunde fing die Pat. bitterlich ohne Schluchzen zu weinen an. Die Muskelunruhe war viel ausgesprochener, als sonst der Fall zu sein pflegt. Der Grund war schlechthin, dass die Pat. von einer neuen Wärterin in einer ungewohnten Weise im Stuhle placirt worden war. Ausserdem wurde sie auch noch von Angst und Unruhe gequält. Der Speichel floss ans dem Munde herans und eine klare seröse Flïssigkeit ans der Nase. Sobald man die Lage der Pat. geändert hatte und sie anf den alten Platz wieder gekommen war, wurde sie ruhio. Die Intelligenz scheint abzunehmen.

21. Oct. Der Schnupfen dauert fort. An gewissen Stellen des linken Unterschenkels beginnt eine klare seröse Flüssigkeit hervorzusickern. Urinmenge wechselnd zwischen 400 und $750 \mathrm{ccm}$ pro 24 Stunden.

Die Thyreoidintabletten werden ausgesetzt and Diuretin viermal täglich ordinirt.

29. Oct. Das Diuretin wird ansgesetzt. Für Pat. wird Infus. digital., 1 Esslöffel $3 \mathrm{mal}$ tägl., ausgeschrieben. Der Schnupfen hat jetzt fast anfgehört.

Am linken Unterschenkel sickert immer noch Flüssigkeit hervor und zwar täglich eine recht erhebliche Qnantität. Die Unterschenkel noch voluminöser als vorher. Haut an denselben röthlich, glänzend, durchsichtig. Das Oedem ist nunmehr etwas weicher geworden, so dass man bisweilen bei Palpation bleibende Eindrücke erzengen kann.

Die Maasse rings um die Unterschenkel sind jetzt die nachstehenden:

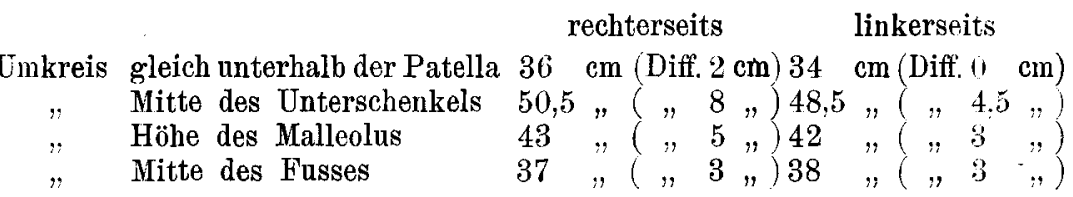

Demnach haben beide Unterschenkel an Dicke zugenommen, der rechte am stärksten. Seit einigen Wochen hat Pat. nicht einmal mit Unterstützung zu gehen vermocht. Bei heute vorgenommener Blutuntersuchung betrug der Hämoglobingehalt (nach Fleischl bestimmt) 75. Zahl der rothen Blutkörperchen pro cbmm 4400000.

31. Oct. Die Pat. bleibt für weitere Beobachtung in der Klinik.

\section{Epikrise.}

Der jetzt vorgetragene Krankenbericht bietet nach meiner Ausicht viel Interessantes.

Der ganze Verlauf der Krankheit ist ein derartiger, dass man sich in der Hauptdiagnose, Paralysis agitans, nicht gut irren kann. Der Verlauf ist aber keineswegs ein typenhafter. I)ie sehr verdickten Unterschenkel und Füsse, deren ganze Configuration, die dicke infiltrirte Haut, welche den Fingerdruck nicht festhält, dies Alles deutet darauf hin, dass es sich hier um eine Art von Elephantiasis handelt. Diese Erscheinung tritt aber unter sonstigen Verhältnissen nicht in Begleitung der Paralysis agitans auf, sondern muss als eine seltene 
Combination aufgefasst werden. Weiterhin kommen bei unserer Patientin verschiedene Symptome sehr sonderbarer Art vor. Vor Allem ist ihr eigenthümliches Aeussere zu erwähnen. Die Züge sind in den letzten Jahren so rauh, Nase, Backen, Kinn und Lippen so dick geworden. Unterhalb der Augen, die etwas kleiner geworden sind, hängt die Haut wie kleine Säckchen herunter. Die Lider erscheinen mitunter in gewissem Grade angeschwollen. Die Haut fühlt sich an vielen Stellen des Gesichts dick und infiltrirt an. Dies Alles lässt gleich den Gedanken an Myxödem entstehen.

Die Patientin sieht im Gesichte myxödematös aus, dies lässt sich nicht leugnen. Forscht man in der jetzt angedeuteten Richtung weiter, erfährt man, dass die Haare auf dem Kopfe so dünn geworden sind, dass hie und da dieses sogenannte "Oedema fugax" vorgekommen ist; ausserdem ist die Stimme sehr rauh geworden. Zudem kommt, dass die Pat. von einem intensiven Kältegefühl im Körper heimgesucht worden ist, welche Erscheinung einer ihrer früheren Aerzte mit dem Namen „Nervenfrost" bezeichnet hat. Die Intelligenz nimmt merkbar ab. Dies Alles sind die Symptome nicht der Paralysis agitans, wohl aber des Myxödems.

Die Symptome der beiden Krankheiten haben sich, wie aus der Anamnese hervorgeht, so zu sagen nebeneinander entwickelt, ohne dass man gerade behaupten könnte, die eine Krankheit sei in die andere übergegangen. Dem oben Gesagten zufolge halte ich mich für berechtigt, folgende Diagnose zu stellen: Paralysis agitans und Myxödem oder vielleicht besser: Paralysis agitans myxoedematosa.

Hinsichtlich des Morbus Basedowii sind ähnliche Uebergangsformen zum Myxödem von mehreren Autoren beschrieben worden. Möb i u s schreibt hierüber Folgendes in seiner Arbeit: „Die Bas ed ow'sche Krankheit" (Nothnagel's ,Spec. Pathologie und Therapie"): Durch diese Auffassung und durch die Kenntniss der Fälle von Verbindung wirklichen Myxödems mit Basedow'scher Krankheit wird ein Licht geworfen auf manche Symptome, die in späteren Stadien der Basedow schen Krankheit vorkommen. Ich meine damit die derben Oedeme; die sulzigen Anschoppungen, von denen Basedow spricht, die skleromartige Verdickung der Haut, die allmählich sich entwickelnde Stumpfheit und Gedächtnissschwäcbe, das Ausfallen der Haare, die Schrumpfung der Brustdrüsen und der weiblichen Geschlechtstheile, bestimmte Gelenk- und Knochenerkrankungen. Das sind Myxödemsymptome. Insbesondere scheinen zwischen dem umschriebenen Oedem der Bas e d ow Kranken und dem eigentlichen Myxödem Uebergangsstufen vorhanden zu sein. Wie im Verlaufe der Basedow-Krankheit Myxödemsymptome auftreten können, so. werden zuweilen beim Myxödem einzelne Ba- 
sedow-Symptome beobachtet. Es ist ersichtlich, dass diese ganzen Verhältnisse bedeutungsvoll sind."

In diesem Zusammenhang will ich noch einen Parallelfall referiren von einer Basedow-Kranken, welche ähnliche Miyxödemsymptome wie die oben erwähnten aufwies (Maladie de Basedow avec myxoedem, von Sollier beschrieben: Revue de Médecine XI, 12. p 1000, Jahrgang 1891), und bediene ich mich eines Referats von Möbius (Schmidt's Jahrbücher) ${ }^{1}$ ):

„Eine 39 jährige Frau war nach Jahren ehelichen Unglücks vor 2 Jahren erkrankt. Erst Schmerzen und Steifheit der Hände. Dann Anschwellung auf den Handrücken. Dann Schmerzen und Anschwellung der Knie, erst vorübergehend, später dauernd. Reizbarkeit und Weinerlichkeit. Schlaflosigkeit. Hitzegefühl mit zeitweiliger Steigerung der Körperwärme. Trockener Husten. Durchfall. Amenorrhoe. Doppeltsehen beim Blicke nach oben. Allgemeine Schmerzhaftigkeit und zunehmende Unbeweglichkeit. Polyurie und Pollakiurie; nie Albuminurie.

Als S. zuerst die Kranke sah, sass sie zusammengebückt, regungslos in einem Stuhle und zwar zu jeder Bewegung unfähig. Der Ausdruck des Gesichtes war stumpf. Am meisten fiel eine ganz enorme Anschwellung des Körpers auf. Die unförmlichen Hände und Finger waren halb gebeugt. Jede passive Bewegung war schmerzhaft. In den Gelenken, die offenbar verdickt waren, Krachen. Starkes Oedem, das den Fingerdruck nicht festhielt. Normales Aussehen der trockenen Haut. Die Beine waren wie Elephantenbeine. An den Unterschenkeln war die Haut durchscheinend und der Fingerdruck hinterliess eine Grube. Das Oedem war Abends stärker als früh. Auch Gesicht, Hals und Rumpf waren geschwollen. Auf der Haut zahlreiche Pigmentflecken. Geringer Exophthalmus. Die Augen waren fast immer halb geschlossen. Herzthätigkeit regelmässig, 110-120, bei Erregung 160. Starkes Zittern. Während der Anfälle' von Hitzegefühl $38,1^{\circ}$. Verminderung des Widerstandes der Haut gegen den galvanischen Strom. Allmähliche Besserung. Die Kranke lernte allmählich wieder stehen und gehen. Das Oedem nahm stetig ab. Der Hautwiderstand nahm zu. Als das Oedem zum grossen Theile geschwunden war, konnte man deutlich fühlen, dass die Schilddrüse sehr klein und etwas hart war. Die Gelenke waren schlaff geworden, so das die Gelenkflächen von einander entfernt werden konnten. Der Urin blieb immer normal und war arm an festen Bestandtheilen."

1) Um grössere Uebersichtlichkeit zu erlangen, habe ich diejenigen Symptome, welche beiden Fällen gemein sind, gesperrt drucken lassen. 
Am Schlusse dieses Referats fügt Mö bi u s noch folgende Worte hinzu: „Da hier Morbus Basedowii und ein myxödemartiger Zustand in Verbindung waren, könne man annehmen, dass entweder die Erkrankung, bez. Atrophie der Schilddrüse an Beidem schuld gewesen sei, oder dass ausnahmsweise die Based ow'sche Krankheit nicht mit Hypertrophie, sondern mit Atrophie der Schilddrüse einhergehe und dass nur diese das Hinzutreten des Myxödems bewirke. S. entscheidet sich für die zweite Annahme."

\section{$\mathrm{Nachschrift.}$}

Gleich nachdem ich meine Epikrise beendet, hat Herr Docent Petrén freundlichst meine Aufmerksamkeit auf einen kleinen (achtseitigen) Aufsatz von Frenkel gelenkt, welcher soeben im diesjährigen Bande der Deutschen Zeitschrift für Nervenheilkunde veröffentlicht worden ist. Derselbe trägt den Titel: „Die Veränderungen der Haut bei Paralysis agitans." Die Auffassung, die in diesem Aufsatze zum Ausdruck kommt, ist ganz dieselbe wie die meinige, weshalb ich mir erlaube das anzuführen, was Verf. zum Schluss in Betreff der Pathogenese der fraglichen Krankheit äussert:

„Zum Schluss möchten wir uns noch einige Bemerkungen in Betreff der Pathogenese dieser uns in der Klinik so wohl bekannten und pathologisch-anatomisch so gänzlich dunklen Krankheit gestatten. Mit der Auffindung einer constanten Veränderung der Haut und mit der damit gegebenen Möglichkeit, einen Theil der subjectiven und objectiven, auf das Nervensystem direct hinweisenden Symptome auf diese Veräuderungen selbst zu beziehen, verliert die an und für sich schlecht fundirte Anschauung, dass wir es mit einer Erkrankung des Gehirns oder Rückenmarks zu thun haben, noch mehr an Wahrscheinlichkeit. ...

Mit dieser Anschauung aber, dass nämlich zwar das Nervensystem eine Rolle in der Pathogenese spielt, dass aber zur Erklärung der Symptome selbst die Annahme einer Läsion des Centralnervensystems im Sinne einer der uns bekannten organischen Erkrankungen desselben nicht zulässig ist, reiht sich die Paralysis agitans denjenigen Krankheiten an, welche wie der Morbus Basedowii, das Myxödem etc. in einer krankhaften Störung des Chemismus jm weitesten Sinne ihren letzten Grund haben. Vielleicht ist, was die Muskelfaser alterirt, bei der Parkinson'schen Krankheit dieselbe Schädlichkeit, welche den'Verlust der Elasticität der Haut und der Verdickung verschuldet. ${ }^{1}$ ) Dass eine solche Anschauung neue Aus-

1) Von Frenkel selbst gesperrt.

Deutsche Zeitschr. f. Nervenheilkunde. XIX. Bd. 
sichtspunkte für die Forschung eröffuet, nachdem die Sterilität der stets negativ ausfallenden anatomischen Untersuchung des Nervensystems für diese Krankheit feststeht, ist klar. Mit einer solchen Anschauung erwacht aber auch die Hoffnung, zu der wir in unseren Tagen mehr als je berechtigt sind, dass auch die Therapie der Paralysis agitans aus einer symptomatischen eine ätiologische werden kann."

Sie müssen gestehen, m. H., es sieht wahrhaftig nicht so aus, als liege nur ein blosser Einfall der Auffassung zu Grunde, für die ich oben eingetreten bin. Oder wie will man sich sonst erklären, dass drei verschiedene Personen an weit entfernten Orten aus verschiedenen Gründen und von einander unabhängig zu einer ziemlich bestimmten Auffassung von dem Wesen der betreffenden Krankheit gelangen? Glaubt man etwa, es liege kein Grund hierfür vor? Dies lässt sich schwerlich denken. Die nächste Zukunft wird meiner Ansicht nach an Untersuchungen in Betreff der Pathogenese der Paralysis agitans sehr reich werden.

\section{Auszug aus dem Obductionsprotokoll.}

Kurz, nachdem das Manuscript zum Druck gegeben worden war, verstarb die Patientin in der Klinik. Der Zustand war im Ganzen bis zum Tode unverändert, welcher am 9. März 1900 nach einigen Tagen durch ein Brustübel eintrat (Bronchopneumonie).

Die Patientin wurde am folgenden Tage obducirt, und das Obductionsprotokoll, vom Herrn Docent Dr. Wadstein aufgenommen, ist von sehr grossem Interesse.

Caroline D., Wittwe, 54 Jahre. Gestorben am 9. März 1900, obducirt am 10. März.

Die Leiche von mittlerer Länge, der Rücken mehr als normal gekrümmt, die Schultern hervorgeschoben. Das Manubrium sterni eingeschwenkt, das ganze Corpus dagegen convex, wodurch die Seitenansicht des Brustbeins eine schwach S-förmige Krümmung anfweist. Demzufolge erhält die obere Hälfte des Brustkastens die Form einer schräg nach vorn bis zur Höhe der vierten Rippe herabfallenden Ebene.

Unterhalb dieser Stelle besitzt der Brustkasten die für das Emphysem charakteristische Fassform. Die Beine von eigenthümlicher Form, oben ziemlich schlank und schlaff, unten dick, angeschwollen, wodurch die ganze Extremität ein klöppelförmiges Aussehen erhält. Die Oberschenkel messen in Umkreis beiderseits $34 \mathrm{~cm}$. Gleich unterhalb der Tuberositas tibiae misst der Umkreis rechterseits $33 \mathrm{~cm}$, linkerseits 35 , auf der Mitte des Unterschenkels wieder rechts 40 , links $46 \mathrm{~cm}$, oberhalb der Malleolen rechts 35 , links $34 \mathrm{~cm}$. Anf der Mitte des Fusses misst der Umkreis rechts 33 , links $36 \mathrm{~cm}$. Fettpolster und Musculatur überall schwach entwickelt. 
Die Haut oberhalb der Kniee am ganzen Körper weich und nachgiebig; unterhalb der Kniee ist sie hingegen eigenthümlich grobcarrirt und bekommt dadurch ein matratzenähnliches Aussehen; am linken Bein ist sie von hellrother Farbe. Rings um die Malleolen ist die Haut wie warzig; die Warzen hanfsamen- bis erbsengross. Sie sind ziemlich weich und können bei Druck zum Schwinden gebracht werden; die Haut ist dann an dieser Stelle fein gerunzelt. Die Form der Füsse und Unterschenkel ist eben die für die Elephantiasis charakteristische.

Das Herz ist ansserordentlich schlaff; die Fettauflagerung ziemlich stark. Die Musculatur der rechten Kammer erscheint als ein schmaler, brauner Streifen; die Wand $7 \mathrm{~mm}$ dick, besteht fast ausschliesslich aus Fettgewebe mit spärlichen, querverlaufenden, etwas bräunlichen Zügen als Reste der Musculatur. Betreffs der Ostien und Klappen ist nichts zu bemerken. Die linke Kammer ist ein wenig erweitert, die Musculatur von etwa $1 \mathrm{~cm}$ Dicke, im Schnitt trocken und gelbflammig. Das Balkengewebe atrophisch, abgeflacht. Die Mitralöffnung lässt mit Leichtigkeit drei Finger hindurchtreten. Die Klappensegel lang und dünn. In der Aorta kommen zahlreiche gelbe, oberflächliche Degenerationsherde vor. In der Höhe der Art. subclavia findet sich eine zehnpfenniggrosse Kalkscholle. Ausserdem gewahrt man im unteren Theile der Aorta verschiedene, ziemlich niedrige Erhebungen, welche auf dem Durchschnitt als halbmondförmige Verdickungen der Intima erscheinen.

In den Lungen verschiedene bronchopneumonische Herde.

Die Milz ist ziemlich gross und fest. Die Kapsel leicht abziehbar, scheint etwas gespannt za sein. Die Trabekeln treten deutlich hervor.

Die Nieren von normaler Grösse. Die Kapsel lässt sich mit gewöhnlicher Leichtigkeit abtragen, aber die obere Fläche der Nieren ist nicht ganz glatt und eben, sondern mit zahlreichen seichten Einbnchtungen und flachen Erhebungen versehen und giebt den Anschein einer im Werden begriffenen Schrumpfniere. Die Rindensubstanz schwillt nicht. Die Consistenz fest. Glomernli ziemlich deutlich. An mehreren Stellen kleine weissliche, feste Knötchen (Fibrome).

Beim Versuch, das Gehirn herauszunehmen, stellt sich heraus, dass das Kleinhirn an dem lateralen Theil der Pars petrosa des Schläfenbeins „angewachsen" ist. Das Gehirn lässt sich nur mit Mühe von dieser Stelle abpräpariren. Nachdem das Gehirn herausgenommen worden ist, nimmt man am linken vorderen Rande des Kleinhirns eine mehr als wallnussgrosse, feste, rundliche Geschwulstbildung wahr, welche in der Substanz des Kleinhirns wohl abgegrenzt liegt und sich leicht herausschälen lässt. Sie ist sehr fest, weshalb tiefere Einschnitte als $1 \mathrm{~cm}$ nicht erzielt werden können. Beim Durchsägen leistet sie einen ähnlichen Widerstand wie Knochen und weist eine radiäre Structur anf. Wenn man die Schnittfläche an der Dura untersucht, gewahrt man, dass die Geschwulst mit einem mehr als zehnpfenniggrossen fibrösen Stiel von der Dura ausgewachsen ist. Die Dura lässt sich an dieser Stelle mit gewöhnlicher Leichtigkeit abtrennen. Im Uebrigen bietet weder das Gehirn noch Rückenmark makroskopisch etwas Abnormes.

Die Schilddrüse ziemlich klein, insbesondere der rechte Lappen, welcher nur $31 / 2 \mathrm{~cm}$ in der Länge misst. Der linke Lappen ist $4 \frac{1}{2} \mathrm{~cm}$ lang. Ein eigentlicher Zwischenlappen fehlt. Die Consistenz des rechten Lappens 
ziemlich fest. Der Querschnitt zeigt nirgends ein für die Schilddrüse normales Aussehen, indem die Colloidfollikel nur hie und da, und zwar nur andeutungsweise, zum Vorschein kommen.

Die Farbe ist weisslichgrau, mit zahlreichen röthlichen Zügen. Hier und dort treten kleinere, gelbliche Partien auf. Bei der mikroskopischen Untersuchung, welche aber noch nicht völlig beendet ist, zeigt dieser Lappen einen Reichthum an bindegewebigen Fasern; das Follikelepithel ist im Allgemeinen niedrig, und das Aussehen des Follikelinhaltes von dem normalen bedeutend abweichend. In dem Verbindungsstück zwischen den beiden Lappen liegt eine bohnengrosse, von der Umgebung deutlich abgegrenzte, Partie von etwas hellerer Farbe (Adenom), in deren Mitte eine hanfsamengrosse, gelbliche Stelle vorkommt.

Der linke Lappen zeigt im Allgemeinen normaleres Aussehen als der rechte. Ungefähr in der Mitte finden sich zwei dicht neben einander gelagerte, bohnengrosse, gut abgegrenzte cystische Bildungen mit colloidem Inhalt.

Hypophysis cerebri ohne Veränderung.

Anlässlich des Sectionsergebnisses seien mir hier nur einige burze Bemerkungen vergönnt.

Was zuerst die Geschwulstbildung in der linken Kleinhirnhemisphäre betrifft, so scheint es mir nicht sehr wahrscheinlich, dass dieselbe an diesem ganz verwickelten Krankheitsbilde die Schuld tragen könnte. Allerdings muss eingestanden werden, dass in der Literatur Fälle beschrieben worden sind, wo der Paralysis agitans ähnelnde Krankheitszustände bei Tumoren in der Hirnschenkelgegend vorgekommen sind, aber nie hat man gehört, dass eine wallnussgrosse Geschwulst in der einen Kleinhirnhemisphäre eine so ausgesprochene Paralysis agitans, wie im vorhandenen Falle, würde hervorrufen können. Zudem hat diese Geschwulst keines von den Symptomen hervorgerufen, die sonst Kleinhirntumoren zukommen. Ich bin deshalb am meisten geneigt, diesen Sectionsfund als etwas Accessorisches aufzufassen, was mit dem klinischen Krankheitsbilde, das ich oben geschildert, nichts gemein hat.

Ebensowenig kann ich den geringen Veränderungen an den Nieren etwaige grössere Bedeutung beimessen.

Es bleibt also nur die Schilddrüse, welche sich wirklich verändert zeigte, und zwar gerade so, wie ich es vom klinischen Standpunkte aus angenommen hatte. Ich hatte mich ja vor dem Verein der Aerzte in Lund, als ich meine Pat. demonstrirte, dahin ausgesprochen, dass sie in gewissem Grade an Myxödem leide, welche Krankheit in diesen Gegenden nicht besonders oft vorkommt. Ein Myxödem setzt ja nach unserer jetzigen Auffassung eine Schrumpfung oder Insufficienz der Schilddrüse voraus.

So verhielt es sich auch mit dieser Patientin. Zwar habe ich noch nicht Gelegenheit gehabt, dieses Organ, das aufbewahrt und sorg- 
fältig fixirt worden ist, eingehender zu untersuchen, aber so viel steht schon fest, dass eine Bindegewebswucherung wenigstens im rechten Lappen stattgefunden hat. Dieser war nicht unbedeutend kleiner, als es sonst der Fall zu sein pflegt. Nicht einmal der linke Lappen war von ganz normaler Grösse.

Die mikroskopische Untersuchung der Glandala thyreoidea und eines Theils der Haut vom rechten Unterbein ist im pathologischen Institut zu Helsingfors unter Aufsicht des Herrn Professors E. A. Homén ausgeführt worden, dem ich hiermit meinen besten Dank ausspreche.

Die erwähnten Organtheile habe ich theils in 10 proc. Formollösung, theils in absolutem Alkohol, theils in Sublimat fixirt und zum Vergleich dieselben Theile normaler Organe aufbewahrt und auf dieselbe Weise behandelt. Folgende Färbungsmethoden sind zur Anwendung gekommen: Hämatoxylineosin, van Gieson, Biondi-Heidenhain's Triacid, Eisenhämatoxylin und für die Haut ausserdem noch Weigert's und Malory's Elastinfärbung und Unna's Färbung der Plasmazellen.

Lobus dexter der Glandula thyreoidea zeigt iiberall ein gleichartiges Aussehen. Man findet besonders perivasculär, aber anch mehr diffus eine manchmal ziemlich bedeutende Wucherung des Bindegewebes. Dieses Bindegewebe ist nicht besonders zellenreich, vielmehr homogen structurlos, was die gefärbten und ungefärbten Präparate sehr deutlich zeigen. In diesem Gewebe befinden sich hier und da stark lichtbrechende Körnchen verschiedener Grösse, fast nie so gross wie ein Zellkern; nicht selten sind Uebergangsformen zwischen jenen und den Zellkernen zu finden. Diese Körnchen empfangen begehrlich Hämatoxylin (Delafield's). An den Eisenhämatoxylinpräparaten erscheinen sie intensiv schwarz gegen die heller gefärbte Umgebung. Auch Carmin fürbt sie. Untersucht man dann die Alveolen genauer, so findet man sie vielerseits sehr verändert, bald sind sie cystisch erweitert und confluirend, bald degenerirt. Fig. 1 anf Tafel III. IV giebt eine gute Uebersicht davon.

Die intensiv schwarzen Flecke sind degenerirende Alveolen. Das Bild ist von einem Eisenhämatoxylinpräparat genommen. Auf einigen Stellen (b, b, b) sieht man hellere Partien structurlosen Bindegewebes. In die Augen fallend ist der grosse Gefässreichthum, trotz der augenfälligen Atrophie des Lobus.

Wenn man bedenkt, dass der rechte Lobus viel weniger umfangreich als gewöhnlich ist, die Intercellularsubstanz aber bedeutend vermehrt und die Blntgefässe nicht weiter reducirt sind, so geht daraus ohne Weiteres hervor, dass eine bedeutende Reduction der eigentlichen Drïsensubstanz des Alveolarepithels vor sich gegangen ist. Es fragt sich, auf welche Weise diese Reduction denn geschieht? Die Antwort erhält man bei genauer Untersuchung eines wohlgelungenen Präparates verschiedener Theile. Figg. 2, 3 und 4 auf Tafel III, IV sind nach demselben Präparate gezeichnet. Auf einigen Stellen (Figg. 2 und 3) scheint das Bindegewebe die Alveolen zu zersprengen, wodurch einzelne Zellen und Zellgruppen hier und da zerstreut liegen. Diese Zellen verschwinden früher oder später; ehe sie aber 
völlig verschwunden sind, sieht man gewöhnlich im umgebenden Bindegewebe eine Anhänfung der ebenerwähnten Körnchen, welche Körnchen nach meiner Ansicht grösstentheils aus Zellen, und zwar am nächsten aus Zellkernen entstehen, welche zerfallen oder degenerirt sind. Möglich ist, dass ein Theil dieser Körnchen ans Pigment besteht. (Ich habe keine Eisenreaction gemacht, da ich es weniger wichtig gefunden.) Die Körnchen werden seiner Zeit in die Circulation aufgenommen und weggeführt. Fig. 2 zeigt ein grosses Gebiet mit solchen einzelnen Zellen und Zellgruppen, die in fast structurlosem Bindegewebe liegen. An den Seiten der Zeichnung finden sich Blutgefässe und eine grössere Alveole. Fig. 3 zeigt uns zwei Alveolen, die im Zerfallen sind; bei $a$ ist das Lumen einer Alveole noch deutlich zu sehen, und auf einem kleinen Gebiet ist etwas Alveolarepithel geblieben. Grösstentheils ist dieses doch zerstört und besteht jetzt aus einer körnigen Masse ohne äussere Abgrenzung; bei $b$ ist der Process viel weiter vorgeschritten. Zwar sieht man eine schwache Andeutung vom Drüsenlumen, aber keine dentlichen Epithelzellen, nur eine dunkle, körnige Masse ist weiter zu entdecken.

Auf anderen Stellen scheinen die Alveolen so zu sagen zu verschwinden, hier aber im Zusammenhang. In Fig. 4 suche ich eine Vorstellung davon zu geben. Der Inhalt solcher Alveolen färbt sich viel stärker als derjenige der übrigen und zwar mit allen benutzten Färbungsmethoden. Dies tritt sogar an ungefärbten Präparaten schärfer hervor. Bei beginnender Degeneration dieser Art scheint das Alveolarepithel anzuschwellen, die innere Begrenzung wird undeutlicher, hier und da sieht man eine. oder mehrere vacuolenähnliche hellere Partien, ebenso einige Kerne oder Kernreste, der Inhalt der Alveolen ist mehr körnig und lässt sich - wie erwähnt wurde - sehr stark mit Eosinroth, mit Eisenhämatoxylin schwarz u. s.w. färben. Mit schwacher Vergrösserung zeigen sich solche Alveolen wie dunkle, von helleren Ringen umgebene Flecken (Fig. 1a). Fig. 4 zeigt dasselbe nnter stärkerer Vergrösserung. Die mit $\mathrm{n}$ bezeichneten Alveolen sind mehr normal, die mit $a, b, c$ und d repräsentiren dagegen schon etwas degenerirte; $a$ und b gehören zu den ersten, früheren Stadien, c und d zu den späteren. Je weiter der Degenerationsprocess fortgeschritten ist, desto mehr fliesst das Alveolarepithel mit seinem Inhalt zusammen, alle Grenzen werden undeutlicher, die Zellstructur wird schliesslich ganz und gar zerstört. Zuerst sieht man, wie eine solche degenerirte Partie auf einem mehr begrenzten Gebiete mit dem umgebenden Bindegewebe wie zusammenfliesst, allmählich schreitet der Process weiter, bis es schliesslich unmöglich wird, eine Alveolarstructur zu sehen. Das Ganze ist in structurlose Interalveolarsubstanz aufgegangen.

Die rothen Streifen, die bei der Section makroskopisch zu sehen waren und welche im Protokolle erwähnt wurden, sind grössere Gefässe.

Der linke Lobus ist dem rechten garnicht gleich. In den oberen und unteren Partien findet man eine beginnende Cystenbildung, schmälere and weniger blutreiche Bindegewebssepta, grössere Drüsenlumina und niedrigeres Alveolarepithel als gewöhnlich. Inmitten des Lobus ist der Process noch weiter fortgeschritten, da liegen viel grössere Cysten nebeneinander und das Ganze hat das Aussehen einer emphysematösen Lunge.

Die Haut ist stark verdickt, was auf reichlicher Entwicklung der Cutis beruht. Gleich unterhalb der Epidermis findet man oft eine offen- 
bare Zellinfiltration und tiefer findet sich ein ziemlich breites Lager eines mehr homogenen structurlosen Bindegewebes, welches von normaler Haut bedeutend abweichende Reactionen giebt. Es nimmt Farbstofte, wie Eosin, Fuchsin u. s. w., weit schwächer zu sich als gewöhnlich und giebt auch in gewissem Maasse andere Farbennuancen. Mit Weigert's Elastinfärbung treten hier keine elastischen Fasern hervor. In den tieferen Lagen der Unterhaut dagegen sind elastische Fasern sehr deutlich zu sehen. Mehrere. Präparate sind gemacht worden und es herrscht constant dasselbe Verhältniss. In den untersten Lagen der Cutis sieht man - doch nicht zahlreich - zersprengte schraubenähnliche Bindegewebshaufen, welche sich beinahe wie Bindegewebe in normaler Haut färben lassen. In den helleren, mehr homogenen Partien zwischen den Fasern des Bindegewebes befindet sich eine nicht unbedeutende Zellanhäufung, oft in Gruppen zerstreut. Erst ziemlich tief unten in der Unterhaut fängt das Fettgewebe an. Tafel III, IV, Fig. 5 a und b sind mit Lupenvergrösserung gezeichnet, um das oben Erwähnte zu verdentlichen; a ist ein Stïck normaler Haut von demselben Theile wie $b$, der meiner Patientin entnommen ist. Beide Präparate sind auf dieselbe Weise fixirt und gefärbt (Biondi's Triacid).

Ich habe die Unna'sche Methode für Färbung der Plasmazellen (in polychromem Methylenblau) benutzt und habe dabei recht zahlreiche Gruppen blaugefärbter Zellen hervorgebracht, welche den Plasmazellen Unna's doch nicht ganz zu entsprechen scheinen. Zum Vergleich habe ich wohlgelungene derartige Präparate aus dem hiesigen pathologisch-anatomischen Institut gebrancht. Zwar haben mehrere Zellen in meinen Präparaten grosse Aehnlichkeit gezeigt, jedoch sind die meisten kleiner als echte Plasmazellen und man vermisst meistens nm den helleren Kern die dunkle, körnige perinucleäre Zone. In den Controlpräparaten normaler Haut treten keine Zellen dieser Art hervor.

\section{Epikrisis.}

Die mikroskopische Untersuchung hat ganz die Richtung genommen, die ich vom rein klinischen Standpunkte aus vermuthete. Es ist wohl ohne Weiteres klar, dass eine Thyreoideadrise der Art, wie ich sie beschrieben, in nicht geringem Grade insufficient sein muss. Die Symptome, die ich als myxödematös gedeutet habe, beruhen also nach meiner Ansicht auf dieser Thyreoidea-Insufficienz. Etwas Anderes ist es, die Paralysis agitans zu erklären. Ich habe ja gefunden, dass der rechte Lobus der Thyreoidea keiner reinen Atrophie unterworfen ist, dazu ist er zu gefässreich. Weiter ist der ganze linke Lobus cystisch verändert. Unter solchen Verhältnissen ist man ja gezwungen anzunehmen, dass das Secret, welches die Drüsen liefern, pathologisch ist; und dass dieses pathologische Secret aus einem so wichtigen Organ, wie die Glandula thyreoidea, bedeutende Störungen im Innern des Körpers und eine dauernde und schwere Nervenkrankheit hervorrufen kann, ist ja nicht unmöglich. Der Chemismus der Thyreoidea ist leider noch nicht bekannt; wir haben noch keine bestimmten mikrochemischen 
Reactionen für dieses Organ. Der Kliniker setzt doch schon jetzt voraus, dass die Thyreoidea unter pathologischen Verhältnissen auf verschiedene Weise secernirt, und es kommt jetzt auf den Morphologen und Mikrochemiker an, verschiedene Reactionen aufzufinden, welche dieses nachzuweisen im Stande wären. Die Secretion der Thyreoidea kann nämlich bei benignen und malignen Formen der Struma (inclusive Morb. Basedowii) nicht gleich sein. Ausserdem giebt es ganz gewiss andere, noch unbekannte Thyreoideakrankheiten (ohne Struma?). 